\title{
Téoros
}

Revue de recherche en tourisme

\section{Le tourisme halieutique et cynégétique en France métropolitaine, entre mise en marché et valorisation sociale des territoires Essai d'identification des facteurs de légitimation}

\section{Frédérick Guyon}

Volume 32, numéro 1, 2013

Tourisme cynégétique et halieutique

URI : https://id.erudit.org/iderudit/1036653ar

DOI : https://doi.org/10.7202/1036653ar

Aller au sommaire du numéro

Éditeur(s)

Université du Québec à Montréal

ISSN

0712-8657 (imprimé)

1923-2705 (numérique)

Découvrir la revue

Citer cet article

Guyon, F. (2013). Le tourisme halieutique et cynégétique en France métropolitaine, entre mise en marché et valorisation sociale des territoires : essai d'identification des facteurs de légitimation. Téoros, 32(1), 47-55. https://doi.org/10.7202/1036653ar

\section{Résumé de l'article}

Le tourisme halieutique et encore moins le tourisme cynégétique n’ont eu, en France, un essor remarqué malgré les actions des acteurs politiques et économiques. Là où la faisabilité technique semble validée, des obstacles de nature sociale et culturelle se rencontrent. Les activités de prédation en France métropolitaine se caractérisent comme étant des activités structurées par des organisations locales, pour des usages sociaux des territoires. Une forte prégnance des phénomènes macrosociologiques se remarque également avec le processus de civilisation des moeurs. La construction des territoires touristiques permettant à une offre de tourisme de prédation d'exister résulte de la concordance de certains facteurs sociaux mais aussi de l'action de structures socioéconomiques. Nous analysons cette mise en tourisme par l'intermédiaire des discours et des offres des promoteurs du tourisme de chasse et du tourisme de pêche. Ces derniers oscillent entre une mise en marché et une valorisation sociale des territoires. Au final, le tourisme rural apparaît comme étant nécessairement un tourisme local, voulu et maîtrisé par les « gens du pays". 


\title{
Le tourisme halieutique et cynégétique en France métropolitaine, entre mise en marché et valorisation sociale des territoires Essai d'identification des facteurs de légitimation
}

\author{
Frédérick GUYON \\ Docteur, maître de conférences \\ Université de Franche-Comté, Laboratoire C3S \\ fredguyon@gmail.com
}

\begin{abstract}
RÉSUMÉ: Le tourisme halieutique et encore moins le tourisme cynégétique n'ont eu, en France, un essor remarqué malgré les actions des acteurs politiques et économiques. Là où la faisabilité technique semble validée, des obstacles de nature sociale et culturelle se rencontrent. Les activités de prédation en France métropolitaine se caractérisent comme étant des activités structurées par des organisations locales, pour des usages sociaux des territoires. Une forte prégnance des phénomènes macrosociologiques se remarque également avec le processus de civilisation des mœurs. La construction des territoires touristiques permettant à une offre de tourisme de prédation d'exister résulte de la concordance de certains facteurs sociaux mais aussi de l'action de structures socioéconomiques. Nous analysons cette mise en tourisme par l'intermédiaire des discours et des offres des promoteurs du tourisme de chasse et du tourisme de pêche. Ces derniers oscillent entre une mise en marché et une valorisation sociale des territoires. Au final, le tourisme rural apparaît comme étant nécessairement un tourisme local, voulu et maîtrisé par les «gens du pays».
\end{abstract}

Mots-clés: Tourisme cynégétique et halieutique, territoire, mise en marché, tourisme local.

S'intéresser aux formes actuelles du tourisme de chasse et du tourisme de pêche, c'est étudier la place et la trajectoire que poursuivent ces formes de rapport à l'espace. Aux interfaces du tourisme et des activités de prélèvement, ce fait social apparaît comme étant une réalité marginale malgré deux éléments. D'une part, les demandes et attentes des touristes pour les territoires ruraux comme lieux de villégiature ne sont plus à démontrer. D'autre part, la France possède le plus grand réseau hydrographique d'Europe occidentale ainsi que des territoires variés permettant divers modes de prédation. Dans le dernier rapport du Conseil national du tourisme en France (Messager, 2011), les activités de chasse et de pêche n'apparaissent pas en tant qu'éléments structurants de cette facette de l'économie. De plus, aucune des études concernant les chasseurs et mettant en avant des profils de pratiquants ne parle des «touristes chasseurs» ou des «chasseurs-touristes» (Union nationale des fédérations départementales des chasseurs, 1988, 1994; Institut National d'Agronomique Paris-Grignon, 1992; Darbon, 1997; CSA : 2006). D'autres analyses font état du même constat de l'absence de touristes pêcheurs étrangers (Lafage, 1992). Ainsi, le tourisme pêche «n'a pas réussi à enrayer le déclin des effectifs de pêcheurs en zone rurale» (Le Goffe et Salanié, 2004 : 16). En plus d'une demande qui semble «marginale», on constate que la pêche en eau douce ne dispose pas d'une offre structurée (Porchier, 2008). Enfin, en ce qui concerne les collectivités territoriales, le tourisme pêche ne semble susciter «actuellement qu'un intérêt limité sur le bassin de la Loire et de ses affluents » (Cabinet Aménagement et tourisme, 2007 : 19).

Néanmoins, des initiatives en matière de tourisme de chasse ou de pêche sont repérables et de plus en plus fréquentes. En effet, les études de faisabilité se multiplient depuis une dizaine d'années (Cabinet A2H, 1997; Conseil général des PyrénéesAtlantiques, 2003; Cardon, 2007; Chopard-Lallier et al., 2007; Cabinet Aménagement et tourisme, 2007; Leroy-Warnier et Caron, 2008; etc.). Elles concluent toutes à des possibilités techniques et administratives de développement touristique.

Le tourisme, en tant qu'activité économique et sociale, traduit un lien organique avec l'espace. Comme nous parlons ici des spécificités du tourisme rural, le milieu «rural», avec ses acteurs et son territoire, apparaît comme un facteur clé dans la compréhension du phénomène. L'écotourisme, qui est en fait 
l'une des formes du tourisme durable qui met l'accent sur la protection de la nature et du patrimoine local, «ne s'est imposé que récemment en tant que solution réaliste de développement permettant à la fois de conserver le patrimoine naturel et culturel des nations et des régions et, du coup, de contribuer au développement durable» (Gagnon, 2010 : 7). Il représente "une composante du tourisme de nature auquel sont associées toutes les activités qui s'effectuent dans l'environnement naturel. Il peut s'agir d'offres de tourisme d'aventure "dur" ou "doux", d'activités physiques de plein air, d'activités contemplatives et enfin d'activités de prélèvement fauniques telles que la chasse et la pêche» (Dupré, 2006 : 54). Sous certaines conditions, les tourismes halieutique et cynégétique peuvent s'intégrer au «tourisme sportif d'action» (Sobry, 2004). Ainsi, on constate depuis les années 1980 une "sportivisation» des discours. La chasse et la pêche occupent, en chiffres cumulés, le septième rang des pratiques sportives des Français (Ministère de la Jeunesse et des Sports, 2001) et des pratiques de prédation (Fabiani, 1982; Guyon et Fuchs, 2009; Callède, 2007).

En analysant les contextes économiques, sociaux et culturels français, nous remarquons que le tourisme cynégétique et halieutique se trouve tiraillé entre des initiatives des territoires et des acteurs locaux « historiques». Au coeur des enjeux apparaissent les pratiquants locaux, les demandes potentielles des voyageurs, les acteurs politiques et touristiques, donc autant d'attentes singulières. Nous cherchons donc à comprendre les facteurs qui influencent la trajectoire particulière que poursuit cette forme de tourisme dont l'origine se situe dans les activités de subsistance pour s'épanouir aujourd'hui parmi les loisirs (culturels et sportifs). Ainsi, nous nous demandons si sous des enjeux touristiques ne se dissimule pas une recherche de valorisation identitaire des humains (Pelosse et Vourc'h, 1982; Traïni, 2004), de leurs activités ancrées sur des territoires et dans des cultures locales, ou si le développement ne répond qu'à un souci économique de mise en marché du territoire rural (Cardon, 2007; Sol, 2007). Ainsi, certains usages du tourisme halieutique et cynégétique par les acteurs (associations, collectivités, entreprises marchandes) semblent poursuivre des finalités plurielles. Malgré les différentes initiatives récentes des acteurs politiques et économiques, ni le tourisme halieutique ni le tourisme cynégétique n'ont eu un essor remarqué en France, signe de l'existence de freins sociaux et culturels forts. Pour vérifier le bien-fondé de ces hypothèses et de leurs conditions d'application à la revue de littératures scientifique et professionnelle, nous associons une analyse des discours et des offres commerciales des organisations et des outils de communication des territoires. Débutant ainsi notre propos par une mise en contexte des activités de prédation en France, nous aborderons ensuite les éléments constitutifs de ces rapports à l'espace, à savoir la place du processus de civilisation dans les rapports à la nature, la patrimonialisation des propriétés des territoires, la «sportivisation» (euphémisation des pratiques, éthique, esprit sportif, aspect ludique, etc.), l'écologisation des rapports à la nature (statut des animaux, usages de l'espace, etc.), la construction des terroirs ainsi que la mise en marché des activités. Enfin, à l'aide de cas concrets, nous montrerons comment s'effectue la mise en tourisme des activités de chasse et de pêche.

\section{Les conditions sociales d'évolution des activités}

Lors des deux parties suivantes, nous montrerons ce que sont les activités de prélèvement ainsi que leur perméabilité aux phénomènes macrosociologiques.

\section{Les activités de prélèvement en France, des activités organisées pour des usages sociaux des territoires}

Les activités de prélèvement reposent sur une même action, celle de «retirer» des éléments de la faune de leur milieu. Pour la chasse, on distingue quatre modes : à tir, à courre, au vol et traditionnelle aux engins. Toutes les autres façons de se procurer du gibier sont interdites. La pêche a pour fin la capture de poissons, de batraciens ou de crustacés. Les types de pêche autorisés sont soit en ligne flottante, soit en plombée, soit à la volante. Au niveau local, deux types de groupements se partagent les territoires de chasse ainsi que leur gestion. Il s'agit des associations communales et intercommunales de chasse agréées et des groupements d'actionnaires ou d'adjudicataires ou des propriétaires de domaines. L'ensemble des associations de chasse (70 000) dépend des fédérations départementales de chasseurs, qui représentent leurs intérêts. Enfin, la fédération nationale regroupe l'ensemble des fédérations départementales. L'article L.222-2 du code rural précise que les associations communales ou intercommunales de chasse agréées ont pour but d'assurer une meilleure organisation technique de la chasse pour permettre aux chasseurs un meilleur exercice de ce «sport». La pratique est sous la tutelle du ministre de l'Écologie, du Développement durable, des Transports et du Logement.

Les droits de chasse et de pêche étant considérés comme des attributs du droit de propriété, la nature des territoires où le droit s'exerce a son importance, car la propriété d'une chose donne droit sur ce qu'elle produit. L'État a la propriété du lit et le droit de l'usage des eaux, donc de pêche, sur son domaine fluvial, mais il se peut que ce droit soit détenu par un particulier. Le droit de pêche de l'État est loué à une association agréée. Toute personne qui se livre à l'exercice de la pêche dans les eaux du domaine public doit justifier de sa qualité de membre d'une association de pêche et de pisciculture ou d'une association agréée (Le Code Rural, article L.236-1). $\mathrm{Au}$ niveau local, les 3901 Associations agréées de pêche et de protection du milieu aquatique (AAPPMA) assurent la surveillance, l'exploitation des droits de pêche, la protection du patrimoine piscicole, le développement des pratiques. La pêche serait le deuxième loisir français derrière le football et le premier loisir de pleine nature (FNPF, 2011).

Aux contraintes spatiales s'ajoutent des limites temporelles liées aux périodes d'ouverture et de fermeture établies en fonction des espèces et des régions. Enfin, pour la pratique, la possession d'un permis est nécessaire et celui-ci est validé pour une «campagne», c'est-à-dire pour une année et pour un territoire. Nous voyons donc que les initiatives touristiques doivent entrer dans ce cadre organisé et réglementé.

Un autre élément primordial nous renseigne sur la place des activités de prédation : la chute constante depuis les années 1960 du nombre de pêcheurs en rivière et de chasseurs. Pour la saison 2008-2009, les pêcheurs ne représentent plus que $2,3 \%$ et $2 \%$ de la population française (ONCFS, 2009; CSPONEMA, 2009) (voir tableau 1). La pêche est associée, pour un 
Tableau 1: Évolution des effectifs de chausseurs

\begin{tabular}{|c|c|c|c|c|c|c|c|c|c|c|c|c|c|c|}
\hline & 1978 & 1979 & 1980 & 1981 & 1982 & 1983 & 1984 & 1985 & 1986 & 1987 & 1988 & 1989 & 1990 & 1991 \\
\hline nombre chasseurs & 2125 & 2064 & 2016 & 1983 & 1989 & 1993 & 1917 & 1863 & 1830 & 1786 & 1749 & 1721 & 1689 & 1659 \\
\hline & 1992 & 1993 & 1994 & 1996 & 1997 & 1998 & 2000 & 2001 & 2002 & 2003 & 2004 & 2005 & 2009 & \\
\hline nombre chasseurs & 1628 & 1602 & 1576 & 1518 & 1496 & 1479 & 1425 & 1407 & 1395 & 1374 & 1358 & 1,320 & 1300 & \\
\hline
\end{tabular}

Source : ONCFS (relevés nationaux de 1978 à 2009).

Tableau 2: Évolution du nombre de pêcheurs

\begin{tabular}{l|l|l|l|l|l|l|l|l|l|l|l|l|l|l|l|l|l}
\hline & $\mathbf{1 9 7 8}$ & $\mathbf{1 9 7 9}$ & $\mathbf{1 9 8 0}$ & $\mathbf{1 9 8 1}$ & $\mathbf{1 9 8 2}$ & $\mathbf{1 9 8 3}$ & $\mathbf{1 9 8 4}$ & $\mathbf{1 9 8 5}$ & $\mathbf{1 9 8 6}$ & $\mathbf{1 9 8 7}$ & $\mathbf{1 9 8 8}$ & $\mathbf{1 9 8 9}$ & $\mathbf{1 9 9 0}$ & $\mathbf{1 9 9 5}$ & $\mathbf{2 0 0 0}$ & $\mathbf{2 0 0 2}$ & $\mathbf{2 0 0 9}$ \\
\hline $\begin{array}{l}\text { nombre } \\
\text { pêcheurs }\end{array}$ & 2530 & 2434 & 2359 & 2349 & 2321 & 2326 & 2266 & 2180 & 2099 & 2045 & 1984 & 1904 & 1825 & 1834 & 2009 & 2000 & 1418 \\
\hline $\begin{array}{l}\text { nombre } \\
\text { assoc }\end{array}$ & 4084 & 4095 & 4110 & 4134 & 4152 & 4151 & 4178 & 4188 & 4209 & 4245 & 4221 & - & - & - & 4167 & - & - \\
\hline
\end{tabular}

Sources : CSP-ONEMA (relevés nationaux de 1978 à 2009).

grand nombre, à une pratique de vacances et reste donc limitée à un contexte et à une temporalité. Cette idée est renforcée par le nombre de cartes «vacances» vendues, qui passent de $0,5 \%$ des ventes totales en 1997 à $19 \%$ en 2009 (cartes journalières ( $15 \%$ ) et vacances ( $4 \%)$ ) (voir tableau 2). Malgré ces nouveaux «produits» qui répondent aux attentes des touristes, l'érosion se poursuit. Trois profils semblent identifiables (Cabinet $\mathrm{A} 2 \mathrm{H}$, 1997) : 47\% sont des pêcheurs terroir sédentaires (la pêche comme un loisir plutôt que comme un motif de départ en vacances), $18 \%$ sont des pêcheurs nouvelle génération, mobiles (pêcheurs sportifs spécialisés dans un type de pêche et dont l'inscription dans les pratiques touristiques «serait de plus en plus forte»), et $26 \%$ sont des vacanciers pêcheurs (leurs attentes sont l'amusement et le repos en famille). Remarquons enfin que les différentes études ne mentionnent à aucun moment des profils de touristes. Ainsi, force est de constater que la demande sociale touristique est mal cernée et ne semble pas entrer dans les préoccupations des commanditaires de ces études.

Ces diminutions sont causées par le non-renouvellement des populations s'adonnant aux activités de prédation sous l'effet des évolutions sociales, culturelles et économiques.

\section{Une forte prégnance des phénomènes macrosociologiques}

La situation rencontrée aujourd'hui résulte de la succession et de la concomitance de phénomènes macrosociologiques qui impliquent les territoires ruraux. Par effet de contagion, ils influencent les activités qui s'y déroulent, les chasses et les pêches, ainsi que la forme particulière qu'ils peuvent prendre : le tourisme halieutique et cynégétique.

\section{Un déficit d'image et d'usage, conséquence du processus de civilisation des mœurs}

Dans les années d'après-guerre, l'agriculture traditionnelle laisse place à une production massive et intensive. Durant cette période, on assiste à une forte augmentation de l'exode rural : les agriculteurs représentent $30 \%$ de la population active, puis
$16 \%$ en 1962 et moins de $4 \%$ aujourd'hui (INSEE, 2009). Leur part parmi les chasseurs chute d'autant. Le recul des terres agricoles qui impacte les chasseurs précède la «désindustrialisation» qui influencera le nombre de pêcheurs (Picon, 1991). La création en 1971 du ministère de l'Environnement marquera une nouvelle étape. De nouveaux textes qui ont permis la "protection des milieux naturels» instaurent pour les citoyens des devoirs envers le patrimoine commun en autorisant ou en prohibant des pratiques, en rationalisant les usages. Cette contrainte symbolique affirme la légitimité de l'action de l'État (Champagne, 1997; Hervieu et Viard, 2001; Darbon, 1997; Elias et Dunning, 1986; Touraine, 1969). Ainsi, selon la loi relative à la chasse de 2000, les pratiques de prédation se doivent d'être raisonnables, rationnelles, insérées dans un système de gestion (plan, lâché, suivi). Les pratiques donnent désormais du plaisir sans heurter les sensibilités (Fabiani, 1989; Dalla Bernardina, 1996; Elias et Dunning, 1986). Enfin, il semble «que moins le rapport à la nature connote une symbolique de violence, plus il est aisément accepté par la population urbaine» (Picon, 1991 : 91). Cela s'exprime plus distinctement chez les pêcheurs, là où la remise à l'eau est possible.

L'intensification et la diversification des usages des espaces ruraux confrontent les communes rurales au nouveau problème de la «fréquentation de leur territoire... par des usagers non résidents» (Boutelet, 1984 : 292). Ainsi, le risque d'une confiscation des espaces naturels est ressenti par les usagers «traditionnels».

\section{Une patrimonialisation des éléments territoriaux}

Les pratiques locales et les coutumes ont laissé des traces dans la vie sociale actuelle. Le nouveau code rural, confirmant les orientations de la loi de 1976, précise que «les espaces, ressources et milieux naturels, les sites et paysages [...] les espèces animales et végétales [...] font partie du patrimoine commun de la nation» (France, 1976). Leur protection, leur mise en valeur et leur gestion sont « d'intérêt général» (France, Article L200-1, 1976). Sous cette mise en patrimoine, l'animal change 
de statut, passant de celui de res nullius (chose qui n'appartient à personne), à celui de patrimoine commun (chose à préserver). Cette catégorie ne va pas s'appliquer à l'ensemble des animaux, certains étant qualifiés de gibier ou de nuisibles. Néanmoins, une nouvelle lecture de la nature s'impose. L'activité de prédation n'apparaît plus nécessairement comme légitime et l'environnement, plutôt comme un lieu de contemplation, un lieu de loisirs urbains (Chamboredon, 1980). On assiste à une valorisation urbaine du passé campagnard, du traditionnel, à un retour des revendications régionales, locales. Ainsi, le tourisme des pays du nord joue «la carte de l'authenticité, de l'identité, de la découverte du patrimoine et de la culture» (Charles et Thouément, 2007 : 37). Les offres halieutiques et cynégétiques se structurent en s'appuyant sur des éléments du "patrimoine naturel», du "patrimoine construit et bâti», du patrimoine «de terroir», regroupant les savoir-faire et les manières d'être, c'est-à-dire les «traditions». Grâce à ces nouveaux objets touristiques, la patrimonialisation «parait bien dotée d'efficacité pour mettre ou remettre en tourisme des lieux restés à l'écart, ou d'autres devenus partiellement obsolètes» (Sol, 2007 : 175). Le processus de patrimonialisation dans ses rapports avec la chasse et la pêche peut également être exploité sous l'angle de la gastronomie (Bérard et Marchenay, 2000).

\section{Une «sportivisation» des pratiques}

La population qui pénètre le monde de la chasse depuis la fin des années 1970 apporte avec elle de nouvelles attentes et des revendications "plus modernes». La nouvelle image du chasseur, diffusée par la conception urbaine et bourgeoise, plébiscite l'activité comme un loisir qui peut être pratiqué comme un sport codifié (l'esprit sportif envers l'animal, les honneurs, la gestion rationalisée, l'activité désintéressée). La référence au sport traduit «la prise en charge par les chasseurs populaires eux-mêmes d'une nouvelle représentation de la pratique» (Fabiani, 1982 : 320). Pour la pêche, on observe le même processus qualificatif visant à valoriser chez les pratiquants un rapport à l'espace et une manière d'être : «[U]n loisir de pleine nature moderne, un retour aux sources qui correspond aux préoccupations environnementales actuelles» (Fédération nationale de la pêche en France, 2009a: 3).

L'apparition puis le développement des parcours traduisent à la fois la pratique de nouveaux publics ayant rompu leurs liens avec le territoire et demandant à être guidés, et l'artificialisation des milieux et des ressources. Les parcours «no-kill» ou «sans paniers» révèlent l'engouement d'un public pour des techniques plus sportives. La finalité gestuelle semble être prédominante sur la quête de produits consommables. Ces nouvelles pratiques répondent également à l'évolution des milieux naturels. En effet, les tableaux de chasse ou de pêche potentiels apparaissent comme un facteur dans le choix de la destination. Toutefois, sous couvert d'un «tableau» garanti, se dissimule une artificialisation des gibiers proposés. La fin d'un gibier «naturel» combiné à une logique gestionnaire et de régulation participe au désenchantement de la quête (Guyon, 2004). Ce sont autant d'éléments inhibant le développement des initiatives halieutiques ou cynégétiques tout en permettant le maintien d'une forme sportive.

\section{L'idée de durabilité des ressources naturelles}

Dans le cas de la chasse plus précisément, les territoires cynégétiques sont tantôt construits comme un «lieu d'évasion» pour une pratique "esthète», comme une "unité de gestion rationnelle» permettant un contrôle écologique ou encore comme un «patrimoine». Trois figures entremêlées de manière inextricable (Traïni, 2004). Le passage d'une "chasse-cueillette» à une "chasse-gestion", puis à une "chasse écologique» et aujourd'hui à une «chasse durable» peut également se lire comme une recherche de positionnement en référence aux aspirations sociales dominantes, voire une stratégie de défense (Ginelli, 2008; Guyon et Fuchs, 2009). Ainsi, les discours des défenseurs des pratiquants résonnent de ces références. Nous pouvons ainsi lire que les chasseurs «doivent veiller à ce que le développement du tourisme cynégétique [...] soit durable, c'est-à-dire bénéfique à la conservation de la faune, de la nature et au développement des populations locales» (Fédération nationale des chasseurs, 2008). Les discours installant les pratiques dans des attitudes écologiques et durables sont le signe de la prise en compte par les instances représentatives des pratiquants, de cette nécessité du positionnement parmi les loisirs responsables et soutenables.

\section{Une localisation des liens en réponse à une globalisation des échanges}

La «culture locale» se trouve définie par des pratiques qui apparaissent comme singulières, car propres à un espace donné, et développées et maintenues par des agents eux aussi pensés comme singuliers. Selon les territoires et selon les cultures de référence, certaines pratiques, certaines quêtes seront valorisées. C'est de l'animal que l'on tire des trophées. Pour qu'un élément puisse être mis en patrimoine, il nécessite au préalable une présence remarquée (Bérard et Marchenay, 1998). Ensuite, "ces attracteurs déterminent une dynamique d'appropriation qui confère un "sens" géoanthropologique à l'émergence des territoires touristiques [...] et déclenchent ainsi la dynamique d'appropriation du potentiel d'attractivité» (Gagnon, 2007 : 11). Comme le consommateur est de plus en plus éloigné des modes de production, il recherche des produits locaux qu'il mythifie. Les activités de prédation par le lien nécessaire au territoire et aux habitants «ancrés» participent à la constitution des terroirs. Ces derniers constituent donc un des piliers des offres touristiques rurales.

\section{Une mise en marché des activités halieutiques et cynégétiques}

Sous l'effet des décisions politiques et des conjonctures économiques, les activités semblent devoir se «moderniser» pour proposer une nouvelle offre et accueillir de nouveaux publics. Depuis la loi sur le développement des territoires ruraux de 2005 qui apporte des outils favorisant l'attractivité des territoires ruraux, des activités de chasse peuvent être cédées à titre onéreux par les sociétés de chasse. De plus, les politiques incitatives nationales et européennes en matière de développement rural et de tourisme vert «ont fait comprendre que le milieu rural possédait des ressources propres à intéresser les citadins, et que des retombées financières non négligeables étaient induites» (Actéon, $1998: 4$ ). Le tourisme cynégétique 
et halieutique est «à la recherche de distinction, c'est-à-dire de l'acquisition de capital culturel nécessaire à l'affirmation individuelle et à la reconnaissance sociale» (Grenier, 2009 : 9). Le tourisme halieutique et cynégétique doit s'employer à la production d'une image de marque valorisante susceptible de permettre à ses adeptes de satisfaire à leurs recherches de pratiques, de territoires et de cultures locales.

Ainsi, nous repérons des changements sociaux qui exercent une influence sur le développement du tourisme de pêche et de chasse. En effet, les évolutions du rapport au territoire naturel et au monde animal, en relation avec son changement de statut, la patrimonialisation de certains éléments locaux et la constitution des terroirs, la «sportivisation» des activités de prédation, l'instauration d'attitudes écologiques et durables et enfin la mise en marché des activités et des territoires modèlent les formes de tourisme proposées.

\section{Des acteurs pour une mise en tourisme de la chasse et de la pêche}

Se pose la question de la mise en tourisme. L'état règlementaire, écologique, social et culturel français étant ainsi constitué, la construction des territoires touristiques permettant une offre de tourisme de prédation résulte de la concordance de certains facteurs mais aussi de l'action de structures socioéconomiques. Nous analysons cette mise en tourisme par l'intermédiaire des discours des promoteurs du tourisme halieutique et cynégétique. La spécificité de l'organisation française fait que les acteurs du marché sont les pratiquants eux-mêmes. Ces organisations appartiennent au secteur privé non marchand, privé marchand et public.

\section{Les initiatives fédérales}

Les fédérations en tant qu'organes de développement, de gestion et de promotion des pratiques affichent leur volonté de voir le tourisme s'émanciper pour pallier notamment la baisse régulière de leurs effectifs. Pour motiver son action, la fédération des pêcheurs annonce que le public est «dans l'expectative d'une réelle offre de tourisme pêche» (Fédération nationale de la pêche en France, 2009b : 1). La réciprocité qui permet de pratiquer la pêche dans 89 départements français doit favoriser «le tourisme pêche»(Ehgo, 2012). De la même manière, la Fédération des chasseurs de l'Isère propose durant la période estivale de partager gratuitement avec eux quelques sessions de comptages et une journée de chasse. C'est une «expérience conviviale pour découvrir l'intimité de la faune iséroise et le rôle primordial de gestionnaire de la faune des chasseurs » (Fédération départementale des chasseurs de l'Isère, 2011). En jouant ainsi sur de nouvelles offres, sur la mise en place d'outils de communication, en entrant ainsi dans une démarche marketing, ces institutions luttent pour le maintien du poids politique et social qui est le leur. Les enjeux sont certes de type économique, mais ils sont avant tout sociaux.

Néanmoins, en tant que représentantes des pratiquants, les fédérations sont confrontées à des enjeux locaux. La construction d'un «vrai produit touristique» semble difficile, car le «mode de gestion ancien» paraît faire obstacle (Cabinet A2H, 1997 : 44). Ainsi, si la faisabilité technique et administrative d'une filière conciliant activités durables et tourisme rural semble réelle, en tenant compte des contextes locaux, les fédérations départementales choisissent de se positionner avec "prudence». En effet, citons en exemple les résultats de cette étude de faisabilité d'un tourisme cynégétique : pour un seul département (sur cinq), "l'accueil des chasseurs extérieurs est apparu comme un véritable désir des chasseurs locaux et de leurs représentants fédéraux» (Chopard-Lallier et al., 2007 : 79). Pour les autres, «une certaine crainte de l'étranger ou de la perte de la maîtrise des territoires, ainsi qu'une réelle inertie des mentalités face à l'émergence du tourisme chasse, opposent [sic] des freins puissants au développement de la filière» (Chopard-Lallier et al., 2007 : 79). Ailleurs, la mise en œuvre pourrait toutefois s'avérer problématique, «en particulier au regard de la valeur identitaire très forte que revêt la pratique de la chasse sur ce territoire» (Leroy-Warnier et Caron, 2008 : 11). La faisabilité technique et administrative n'est pas suffisante, l'acceptation sociale de cette mise en partage du territoire et des ressources reste un facteur limitatif.

\section{Les initiatives marquantes, voire isolantes des associations de pratique}

Les associations locales sont les véritables acteurs de terrain pouvant développer un tourisme de prélèvement. Ainsi, la Fédération départementale de la Manche identifie 22 parcours de pêche portés chacun par une association locale. Cette offre fonctionne comme une segmentation touristique selon des critères d'accessibilité ou de proies potentielles. L'accent donné aux activités va jusqu'à définir un «parcours touristique». Ce dernier se compose d'une offre d'hébergement (un camping), d'une accessibilité et des éléments piscicoles. Cette offre ciblée permet également d'assurer un «contrôle social» des touristes en les guidant vers certains sites de pratique libre, les usagers locaux pouvant conserver l'usage de certains autres. Concernant les activités cynégétiques, peu d'initiatives émanant des Associations communales de chasse agréées (ACCA) sont repérables. L'accueil de chasseurs touristes est possible, même incité par la loi pour les ACCA, mais il reste aujourd'hui essentiellement lié à des impératifs économiques (remboursements des dégâts de gibiers). Le «dilemme associatif» (Auxietre et Jantzen, 1992) s'exprime à propos d'exigences qui peuvent s'avérer contradictoires : entre l'ouverture à l'autre et le renforcement de l'entre-soi. L'accessibilité des «étrangers » aux associations et aux territoires de chasse étant difficile, l'association Phoebus, se définissant comme "intermédiaire de chasse», tente de mettre en relation les chasseurs de grands gibiers extérieurs avec des territoires de chasse bénéficiant de plans de chasse.

\section{Les initiatives des associations de tourisme et de valorisation des territoires ruraux}

On peut repérer trois types d'associations aux finalités différentes. Le premier est représenté par les associations de valorisation du territoire qui émanent des collectivités territoriales, des départements (les comités départementaux du tourisme) et des communes (les offices du tourisme). Le département de la Creuse a structuré sa filière de tourisme de pêche dès 1980 . Toutefois, c'est le schéma départemental de développement touristique élaboré en 2000 par le comité départemental du tourisme et adopté par le conseil général qui classe parmi ses 
priorités la structuration des filières d'activités de nature, dont la pêche. Celle-ci est identifiée comme porteuse d'enjeux en termes économiques, sociaux et environnementaux. La mise en place d'initiatives utilisant les activités de prédation comme des leviers de développement économique concerne des territoires où la ruralité est marquante, voire «isolante». La lutte contre la «désertification» passe par la valorisation touristique du territoire. Ainsi, les activités de prédation, surtout sous leur forme «douce», durable, concourent au développement d'une image de marque territoriale axée sur la nature et sur l'expression de la vitalité du cadre de vie.

Le second type rassemble les associations de valorisation du patrimoine culturel. Elles proposent des activités afin de découvrir les pratiques «traditionnelles». Une sortie en toue cabanée (bateau traditionnel de pêche en Loire) est l'occasion de découvrir «des techniques de pêche, des poissons pêchés en Loire, de relever des nasses et filets " et ensuite de les déguster «autour d'un déjeuner pris en commun, accompagné de produits du terroir» (Association Passeurs de Loire, s. d.). Sans finalité économique, la rencontre et l'hospitalité apparaissent comme étant les éléments moteurs de ces initiatives.

Enfin, le troisième type est illustré par l'association Actéon "Chasse, pêche et tourisme vert au service du développement rural». Créée en 1996, elle applique un principe de pratique «équitable et de redistribution au profit de l'économie locale». Elle fédère les gestionnaires de l'espace rural, agricoles et forestiers, autour de programmes agri-environnementaux financés intégralement ou en partie par des activités touristiques (Actéon, 2012). De même, l'association Pescofi, sous la forme d'un réseau rassemblant 250 professionnels partenaires appartenant aux secteurs du tourisme, de l'hébergement, des loisirs, de la restauration, prépare des séjours «remplis de sensations». Les activités touristiques, sous leur forme associative, sont un levier du maintien d'un dynamisme local, économique.

\section{Les initiatives privées marchandes}

Nous classons les offres de ce secteur en trois catégories : les domaines, les hébergeurs, les organisateurs de séjours.

\section{Les domaines}

Un droit de chasse peut être loué par un particulier à un propriétaire privé ou au domaine public. Le locataire constitue une société avec des partenaires. Si 350 structures privées sont référencées dans l'Annuaire cynégétique français (qui comprend également un annuaire des sites et des organisations de pêche), aucune spécification pour le tourisme n'existe. On peut légitimement se poser la question de l'absence de cette rhétorique : ici le mot touriste n'a pas lieu, il est remplacé par celui de «passionné» ou simplement de pratiquant. Cette offre peut aussi prendre la forme exotique du safari où l'on quête des «trophées d'exception» tout en découvrant les «célèbres richesses culturelles, naturelles, traditionnelles et gastronomiques", dans une "atmosphère très conviviale», afin de "goûter au plaisir et au confort de "la vie de château"! (France Safaris, c2008). Ces offres commerciales construisent un cadre aménagé et sécurisé (Domaine de Pimprez, s. d.) mettant en avant les symboles de l'aristocratie : «domaine», «richesses», "château», «désintéressement», «trophée».

\section{Les hébergeurs}

Certains hébergeurs associent à leurs offres, des pratiques locales pour valoriser leur équipement et ainsi attirer une clientèle spécifique. Par exemple, l'hôtel restaurant du Fronton offre la possibilité de découvrir la chasse à la palombe sur des emplacements qui lui sont réservés. Il propose un forfait journalier comprenant la pension complète et le poste de tir au vol. Pour les passionnés de pêche, «Bruno le patron, pêcheur lui-même, vous prodiguera des conseils bien utiles» (Hotel restaurant du Fronton, s. d.). De même, la fréquentation du camping L'Arize permet de "pêcher la truite dans la rivière dont les eaux fertiles regorgent des variétés les plus diverses» (Camping Arize, s. d.). Les acteurs locaux jouent ainsi le rôle d'interprètes du territoire, d'intermédiaires qui permettent d'accéder aux «meilleurs» sites de pratique, lieux des richesses culturelles. Les labels participent au positionnement des hébergements : ici le label «Pêche Ariège Pyrénées» est attribué à des hébergements, campings, gîtes ruraux, meublés, hôtels, gîtes d'étapes, refuges d'altitude, ainsi qu'à des magasins d'articles de pêche qui répondent à une charte. De même, certaines fédérations de pêche développent la «thématique pêche» en partenariat avec des réseaux d'hébergeurs, type Clévacances ou Gîtes de France.

\section{Les organisateurs de séjours}

Parmi les acteurs privés marchands, nous trouvons les organisateurs de séjours. Par exemple, la société Esprit Sauvage «a été créée pour faire vivre des émotions intenses à des chasseurs, pêcheurs ou simplement globe trotteurs» (Esprit Sauvage, s. d.). Plusieurs types de séjours articulés autour de la chasse ou de la pêche et alliant découverte de la région permettent de "chasser, pêcher comme le font les prédateurs pour atteindre leurs proies, c'est naturel, c'est l'Esprit Sauvage» (Esprit Sauvage, s. d.).

\section{Les initiatives des collectivités publiques ou des services publics}

Le tourisme et l'ensemble des dimensions qu'il englobe prennent place dans les stratégies marketing locales. Ici, «le tourisme halieutique présente de réelles opportunités de développement pour des territoires économiquement fragiles» (Conseil général des Pyrénées-Atlantiques, 2003). Là, l'apport financier des « 4000 touristes pêcheurs sur le secteur franco-suisse peut être estimé à 1220000 euros» (Berthold, 2007 : 5). L'étude de faisabilité pour la mise en place d'un programme de développement rural basé sur «la chasse durable et le tourisme rural» commandée par la région LanguedocRoussillon en 2007 évalue des flux financiers «à hauteur de 46600 à $93200 €$ dans l'hôtellerie, de 78900 à $157800 €$ dans la restauration, pour 196000 à $392000 €$ ». Cela permettrait aussi «de générer ou de maintenir de 200 à 400 emplois sur les territoires ruraux du Languedoc-Roussillon » (Chopard-Lallier et al., $2007: 79$ ). Toutefois, l'étude conclut que la filière «n'a pas le potentiel de constituer une véritable manne financière pour les fédérations», mais qu'elle pourrait «néanmoins générer des emplois et flux économiques sur certains territoires ruraux en contre-saison» (Chopard-Lallier et al., 2007 : 78). Un autre exemple illustre cette implication des collectivités dans le développement économique. Pescalis est une station 
intégrée, dont le maître d'œuvre est la communauté de communes Terre de Sèvre (gérée par une société publique industrielle et commerciale. Si, en 2001, elle était dédiée à la pêche sportive, elle devient aujourd'hui «une station familiale de nature, avec une offre de loisirs qui s'élargit» (Leclercq, 2008 : 51). Le site compte deux résidences de tourisme, un camping, un hôtel et trois gîtes ruraux et une dizaine de prestataires de services. L'investissement total de 16,8 millions d'euros a été porté à $47 \%$ par le public (Europe, État, région, département, collectivité locale) et à $53 \%$ par le privé (hébergement, restauration, activités de loisirs). L'État, par l'intermédiaire de Pôles d'excellence rurale (PER), participe également au dynamisme des territoires ruraux. Le PER intitulé «Accueil chasse et pêche en Lozère : une dynamique de territoire» labellisé en 2006 illustre cela. Il vise «à impulser une nouvelle dynamique autour des activités de pêche et de chasse, tout en conciliant le développement économique, social et environnemental, ainsi qu'en fédérant les acteurs des filières de chasse, pêche, tourisme» (Leroy-Warnier et Caron, 2008 : 4). Par l'intermédiaire des investissements publics, les collectivités et l'État utilisent les richesses territoriales halieutiques et cynégétiques afin de mettre en valeur le territoire. Au final, les retombées espérées sont économiques et territoriales.

\section{L'ONF}

L'Office national des forêts (ONF), établissement public à caractère industriel et commercial (EPIC), propose des possibilités de chasse sur $11 \%$ de la forêt française métropolitaine et $4 \%$ du territoire chassé. La détention du droit de chasse et de pêche a été transférée à l'office lorsque l'État lui a remis en dotation les forêts domaniales. Ces territoires sont exploités sous forme de licences «dirigées» ou «guidées». L'ONF accueille chaque année 100000 chasseurs avec une offre essentiellement concentrée autour de la chasse du grand gibier et dans le cadre des plans de gestion. Aucune offre halieutique n'est proposée.

\section{Un tourisme diffus et des enjeux pluriels}

Le développement du tourisme de pêche et de chasse demeure une question particulièrement sensible dans beaucoup d'associations. Rappelons qu'elles bénéficient (délégation, gestion, etc.) de la totalité des parcours de pêche et des territoires de chasse (hors domaines privés). Par conséquent, elles demeurent les acteurs majeurs et incontournables des politiques de développement touristique. Parmi les freins, évoquons le fait que les administrateurs ne connaissent pas «le marché et ses enjeux économiques» (Ducher, 2008 : 37) ni les «motivations des touristes», et, de ce fait, ont "tendance à les craindre», voyant «en eux des étrangers sans scrupules qui vont venir piller leurs rivières...» (Raimbourg, 2004 : 129) ou leurs territoires, moyen de reproduction du groupe social (Bozon, 1982) ou de l'identité locale. De plus, les modèles de développement utilisés risquent de "dénaturer un patrimoine» (Cardon, 2007 : 67). L'exemple de la chasse en Alsace illustre en partie cette crainte. Ici, là où les flux économiques dépassent les 61 millions d'euros (pour 10000 permis, soit $6100 € /$ chasseur, contre une moyenne nationale de $1350 €$ ), la pratique, induite par le droit local est réservée à des adjudicataires qui représentent une minorité privilégiée, une «catégorie sociale à fort capital économique» (Hell, 2006 : 20). La chasse populaire a disparu en partie au profit des chasseurs étrangers ou aisés. Notons que les relations que les pratiquants entretiennent avec leur territoire diffèrent singulièrement selon leur appartenance à une société locale ou à un groupement d'actionnaires. En effet, là où le but des sociétés de chasse semble être de maintenir le droit de chasse aux membres de la communauté locale, en signifiant leur présence et en rappelant l'existence d'un droit d'usage sur ce territoire (Bozon, 1982), dans le second mode d'organisation regroupant des actionnaires ou des adjudicataires ou des propriétaires de domaines, l'intérêt commercial prend le dessus sur les enjeux sociaux et communautaire. Le tourisme reflète alors une hiérarchie sociale. À cela s'ajoutent les obstacles tels que le déficit d'image et les problèmes de cohabitation avec les activités humaines de loisirs (randonnée, VTT, eau vive (Mounet, 2000)). La question du partage spatial et temporel des territoires reste posée.

Sous couvert d'authenticité, s'exprime la revendication identitaire des territoires. La lutte contre l'anomie sociale locale apparaît de manière sous-jacente. Pour les pratiquants ancrés sur leur territoire, les freins à ces initiatives reposent sur le fait que la solution touristique est moins adoptée par conviction que par résignation : le dernier rempart contre l'isolement, la chute des effectifs et le déclin économique. Cependant, le tourisme «à lui seul ne pourrait pas résoudre toutes les questions de régénération des zones rurales» (OCDE, 1994 : 35). L’usage du tourisme vert est-il «effectivement une réalité susceptible de conduire aujourd'hui les habitants des campagnes, et tout particulièrement ceux impliqués dans l'activité touristique, à penser que leur territoire peut sortir de l'"ornière" de la fragilité grâce à cette voie» (Desmichel, 2000 : 51)? Ces éléments nous amènent à penser le produit touristique par l'intermédiaire du concept du «système touristique localisé» (Hugues, 2008) où les actions ne peuvent être dissociées du système territorial et surtout de son système culturel et des "hauts-lieux» (Debarbieux, 2003) qui le structurent. La valorisation des locaux se réalise par la médiation de leurs pratiques sociales dites traditionnelles, locales, ancestrales, de terroir, etc. Ainsi, la contribution des loisirs sportifs à l'économie touristique s'opère plus «dans le cadre d'un processus de développement territorial global que dans le cadre d'une offre strictement économique s'exprimant en termes de produits» (Bourdeau, 2003 : 225). Les offres et les prestations qui «consomment» la nature ne peuvent se comprendre sans une mise en perspective territoriale globale, c'est-à-dire en tenant compte des enjeux qui traversent les lieux (désertification, image ancienAinsi, si aujourd'hui le tourisme halieutique et encore moins le tourisme cynégétique n'ont eu un essor remarqué, malgré les actions récentes des acteurs politiques et économiques, cela signifie que les freins forts qu'ils rencontrent sont de nature sociale et culturelle. De ce fait, pour les projets se déroulant sur des territoires communaux ou publics, seule l'inscription dans un projet de territoire peut espérer modifier la tendance. Ainsi se pose la question de la place des activités : savoir si la chasse et la pêche sont des atouts (de départ) quand la stratégie touristique est inexistante ou si elles sont de sérieux atouts (supplémentaires) quand l'attraction touristique est déjà présente. Notons aussi que la filière devra certes "avoir un objectif de 
développement local direct (guides, aménagement des milieux, rentrées d'argent pour les sociétés de chasse) et indirect (petit commerce, artisanat, tourisme etc.)» (Chopard-Lallier et al., 2007 : 20), mais surtout prendre corps chez les possédants des droits de pratique. Le développement local durable reposant sur une gestion intégrée des ressources prend ici tout son sens. Le tourisme durable halieutique et cynégétique ne peut se faire sans l'initiative des "médiateurs-interprètes de milieu de vie» (Caire et Roullet-Caire, 2001), sans le savoir-faire des acteurs territoriaux (Gagnon, 2010). Ainsi, certains « acteurs associatifs réussissent à lier conservation, marché et participation des populations locales» (Gagnon, $2010: 4$ ). Pour le Centre national de ressources du tourisme et du patrimoine rural, organisme conseil au service des acteurs locaux, le tourisme rural est nécessairement un «tourisme local, voulu et maîtrisé par les gens du pays, un tourisme de rencontre et de partage, puisant ses arguments dans la richesse des terroirs et la convivialité des habitants» (Association Source, s. d.). Le développement économique n'apparaît pas nécessairement comme étant l'objectif premier, mais comme un élément de l'évolution territoriale possible.

\section{Références}

Actéon (1998) Bilan final de l'étude de l'offre et de la demande cynégétique, Paris : DATAR, Fonds national pour l'aménagement du territoire. $37 \mathrm{p}$.

Actéon (2012) «Actéon", Association Actéon, <http://www.associationacteon.fr $>$, consulté le 15 juillet 2012

Association Passeurs de Loire (s. d.) « Passeurs de Loire, la tradition continue», SARL Passeurs de Loire, <www.passeursdeloire.fr>, consulté le 15 juillet 2012.

Association Source (s. d.) «agriculture.gouv.fr», Ministère de l'Agriculture, de l'Agroalimentaire et de la Forêt, <agriculture.gouv.fr>, consulté le 15 juillet 2012

AUXIETRE, Jean-Pierre et Jean-Marie JANTZEN (1992) «Les termes du défi associatif ", Eaux Libres, vol. 10, p. 34-35.

BERARD, Laurence, et Philippe MARCHENAY (1998) «Terroirs, produits et enracinement, Pour une anthropologie impliquée. Argumentation face aux extrémistes", Association Rhône-Alpes Anthropologie, n 43 spécial, p. 16-17.

BERARD, Laurence et Philippe MARCHENAY (2000) «Le vivant, le culturel et le marchand : les produits de terroir », Vives campagnes. Le patrimoine rural, projet de société, Revue Autrement, nº 194, p. 191-216.

BERTHOLD, R. (2007) «La pêche de loisir entre ville et campagne dans le Doubs», Image de Franche-Comté, Laboratoire ThéMA — CNRS, Université de Franche-Comté, document interne. 1 p.

BOURDEAU, Philippe (2003) «Territoires du hors-quotidien : une géographie culturelle du rapport à l'ailleurs dans les sociétés urbaines contemporaines; le cas du tourisme sportif de montagne et de nature». Diplôme d'habilitation à diriger des recherches, Grenoble : Université J. Fourier-Grenoble 1, Laboratoire Territoires. 267 p.

BOUTELET, Margueritte (1984) «Un régime juridique pour les chemins de randonnée pédestre», R.J.E, Dijon, p. 291-323.

BOZON, Michel (1982) «Chasse, territoire, groupement de chasseurs», Études rurales, $\mathrm{n}^{\circ}$ 87-88, p. 335-342.

Cabinet A2H (1997) Étude pour la valorisation touristique du patrimoine halieutique du département de l'Aisne, France : A2H. 116 p.

Cabinet Aménagement et tourisme (2007) Étude sur le tourisme pêche et ses perspectives de développement à l'échelle du bassin de la Loire et des affluents, Rapport de $3^{\mathrm{e}}$ phase : synthèse du diagnostic et des études de cas, enjeux et conditions de développement du tourisme pêche Stratégie proposée, France : CAT. 40 p.

CAIRE, Gilles et Monique ROULLET-CAIRE (2001) Le tourisme peut-il être un élément de développement durable?, communication au forum «Les enjeux du développement durable», Orcades, <www.tourisme-durable. net>, consulté le 15 juillet 2012.

CALLEDE, Jean-Paul (2007) La sociologie fra nçaise et la pratique sportive, 1875-2005, Pessac: Maison des sciences de l'homme d'Aquitaine. 610 p.

Camping de l'Arize (s. d.) «L'Arize», Camping de l'Arize, <www.campingarize.com/peche/htm>, consulté le 15 juillet 2012.

CARDON, Lucy (2007) «Quelle stratégie "tourisme pêche" en Savoie pour 2007-2013?». Master 1 Mutations des Territoires Urbains et Développement Intégré des Littoraux, Dunkerque : Université du Littoral Côte-d'Opale. 88 p.

CHAMBOREDON, Jean-Claude (1980) «Les usages urbains de l'espace rural : du moyen de production au lieu de récréation ", Revue française de sociologie, janvier-mars, vol. XXI,

CHAMPAGNE, Patrick (1997) «L'environnement, les risques et le champ journalistique, Sur la nature en jeu », Regards sociologiques, ${ }^{\circ} 14$, p. 73-90.

CHARLES, Erwann et Hervé THOUÉMENT (2007) «Le label territorial, facteur d'attractivité touristique. Une étude appliquée à la Bretagne», Téoros, été, p. 33-38.

CHOPARD-LALLIER, F.; L. COURBOIS et J. DOMINGO (2007) Étude de faisabilité pour la mise en place d'un programme de développement rural basé sur la chasse durable et le tourisme rural en Languedoc Rousillon, Fédération Régionale des Chasseurs du Languedoc-Roussillon, Région Languedoc-Roussillon, FEADER. 80 p.

Conseil général des Pyrénées-Atlantiques (2003) Schéma départemental de développement et d'aménagement touristique - Partie 1 : État des lieux du tourisme de Pyrénées-Atlantiques, France : Conseil Général des PyrénéesAtlantiques. $29 \mathrm{p}$.

CSP-ONEMA - Conseil Supérieur de la Pêche-Office National de l'Eau et des Milieux Aquatiques (1978 à 2009) données internes, relevés nationaux.

DALLA BERNARDINA, Sergio (1996) L'utopie de la nature, chasseurs, écologistes et touristes, Paris : Imago. $304 \mathrm{p}$.

DARBON, Dominique (1997) La crise de la chasse en France, La fin d'un monde, Paris : L'Harmattan. 300 p.

DEBARBIEUX, Bernard (2003) «De l'objet spatial à l'effet géographique», DANS L'effet géographique: construction sociale, appréhension cognitive et configuration matérielle des objets géographiques, p. 11-24. Grenoble : MSH-Alpes.

DESMICHEL, Pascal (2000) «Réalité économique et perception sociale du tourisme en milieu rural fragile. Analyse à travers l'exemple de trois territoires, pyrénéens", Revue de géographie alpine, tome 88, nº 3, p. 51-63.

Domaine de Pimprez (s. d.) «Le domaine de pêche du Pimprez : le Domaine de Pimprez vous souhaite la bienvenue sur son site», Domaine de Pimprez, <www.domainedepimprez.com>, consulté le 15 juillet 2012.

DUCHER, I. (2008) «Structuration d'une filière de tourisme de pêche, l'exemple de la Creuse», Espaces, juin, n 260, Pêche et tourisme, p. 37-40.

DUPRÉ, Sophie (2006) «Perception et Représentations géographiques. Un outil pour aménager les forêts touristifiées?», Téoros, été, p. 53-61.

EHGO (2012) «Simplifiez-vous la pêche», Entente halieutique du Grand Ouest, <www.ehgo.fr>, consulté le 15 juillet 2012.

ELIAS, Norbert et Eric DUNNING (1986) Sport et civilisation, la quête du plaisir, Paris : Agora. $392 \mathrm{p}$. 
Esprit Sauvage (s. d.) «Esprit Sauvage : Bienvenue», Esprit Sauvage, <espritsauvage.com ", consulté le 15 juillet 2012.

FABIANI, Jean-Louis (1982) «Quand la chasse populaire devient un sport, La redéfinition sociale d'un loisir traditionnel», Études rurales, n $87-88$, p. 309-323.

FABIANI, Jean-Louis (1989) «La nature, l'action publique et la régulation sociale», DANS MATHIEU, Nicole et Marcel JOLLIVET (sous la direction de), Du rural à l'environnement, La question de la nature aujourd'hui, p. 195-208. Association des Ruralistes Français Edition, Paris : L'Harmattan.

Fédération départementale des chasseurs de l'Isère (2011) Venez chasser avec les chasseurs d'Isère, plaquette publicitaire.

Fédération Nationale de la Pêche en France (2009a) Synergies Pêche: Premières journées nationales d'échanges sur la promotion du loisir pêche et le développement du tourisme halieutique, (13 et 14 octobre 2009, France : Annecy), p. 1.

Fédération Nationale de la Pêche en France (2009b) «Bulletin », Pêche Mag, $\mathrm{n}^{\circ} 4$, juin, p. 3 .

Fédération nationale des chasseurs (2008) «La chasse, acteur du développement durable pour la France et pour l'Europe», 62 Forum Iéna, 29 mai 2008, Conseil économique et social, Info-flash, Fédération Nationale des Chasseurs, $\mathrm{n}^{\circ}$ 17, <www.chasseurdefrance.com>, consulté le 15 juillet 2012.

FNPF - Fédération Nationale de Pêche en France et de protection du milieu aquatique (2011) Adhésion, FNPF, <www.cartedepeche.fr>, consulté le 15 juillet 2012.

France (1976) «Article L.200-1», Code rural, France : Legifrance.

France Safaris (c2008) «Bienvenue chez France Safari», France Safaris, $<$ http://www.francesafaris.com/index-fr.php>, consulté le 15 juillet 2012.

GAGNON, Serge (2007) «Attractivité touristique et "sens" géo-anthropologique des territoires», Téoros, été, p. 3-11.

GAGNON, Christiane (dir.) (2010) L'écotourisme visité par les acteurs territoriaux. Entre conservation, participation et marché, Québec : Presses de l'Université du Québec. 259 p.

GINELLI, Ludovic (2008) Chasse-gestion, chasse écologique, chasse durable... Enjeux autour de l'écologisation d'une pratique en crise, Colloque SFER «Chasse, Territoires et Développement durable. Outils d'analyse, enjeux et perspectives» (25 au 27 mars), France, ENITAC Clermont Ferrand.

GRENIER, Alain A. (2009) «Conceptualisation du tourisme polaire, Cartographier une expérience aux confins de l'imaginaire», Téoros, vol. $28, \mathrm{n}^{\circ} 1$, p. 7-19.

Groupe CSA (2006) Les Chasseurs qui sont-ils?, Sondage CSA Fédération National des chasseurs.

GUYON, Frédérick (2004) «Analyse des rapports pluriels à l'espace "naturel”. Chasseurs, pêcheurs, cueilleurs». Thèse de doctorat de sociologie non publiée, Strasbourg : Université Marc Bloch. 484 p.

GUYON, Frédérick et Julien FUCHS (2009) Se dire «sportif» dans les pratiques de prédation (chasses, pêches, cueillettes) : sens et conditions d'un processus de qualification, Communication au colloque de la 3SLF, France, Nanterre.

HELL, Bertrand (2006) «Morphologie d'un fait social», Terrain, <http://terrain.revues.org/4197>, consulté le 15 juillet 2012.

HERVIEU, Bertrand et Jean VIARD (2001) L'archipel paysan, la fin de la république agricole, La Tour d'Aigues : Éditions De l'aube. 124 p.

Hôtel restaurant du Fronton (s. d.) «Hôtel. Restaurant du Fronton : bienvenue», Hôtel restaurant du Fronton, < hotel-restaurant-fronton.com>, consulté le 15 juillet 2012.
HUGUES, François (2008) «Durabilité des ressources territoriales et tourisme durable : vers quelle convergence?», Géographie, économie, société, vol. $10, \mathrm{n}^{\circ} 1$, p. 64-82.

INSEE (2009) Recensement national de 2009, données en ligne, <insee.fr>, consulté le 15 juillet 2012.

Institut National d'Agronomie Paris-Grignon (1992) Laboratoire de la Faune Sauvage, Union Nationale des Fédérations Départementales des Chasseurs, Institut National Agronomique Paris-Grignon.

LAFAGE, J.-P. (1992) «La pêche en France, un potentiel à libérer », Eaux Libres, vol. 10, p. 19-23.

LECLERCQ, Robert (2008) «Pescalis, station nature», Espace, vol. 261, juilletaoût, Pêche et tourisme, p. 51-52.

LE GOFFE, P. et J. SALANIE (2004) «La gestion associative de la pêche en France : une analyse économique à partir du cas des salmonidés», Bull. Fr. Pêche Piscic., no 375, p. 15-34.

LEROY-WARNIER L. et A. CARON (2008) Tourisme cynégétique, enjeux d'un levier de développement local en devenir. Illustré de l'exemple lozérien, Colloque SFER «Chasse, Territoires et Développement durable. Outils d'analyse, enjeux et perspectives» (25 au 27 mars), France, ENITAC Clermont Ferrand, p. 11.

MESSAGER, Michel (dir.) (2011) «Le poids économique et social du tourisme», Rapport du conseil national du tourisme, session 2010, France : Ministère de l'économie, de l'industrie et de l'emploi. 137 p.

Ministère de la Jeunesse et des Sports (2001) «Le poids économique du sport», Bulletin Stat-Info, no 01-01, p. 6.

MOUNET, Jean-Pierre (2000) «L'impact des loisirs de nature sur le milieu humain", Les Cahiers Espaces, n ${ }^{\circ}$ 67, p. 216-225.

OCDE - Organisation de coopération et de développement économiques (1994) Les stratégies du tourisme et le développement rural, Paris : OCDE. $100 \mathrm{p}$.

ONCFS — Office National de la Chasse et de la Faune Sauvage (1978 à 2009) Données internes, relevés nationaux.

PELOSSE, Valentin et Anne VOURC'H (1982) «Chasse au sanglier en Cévennes», Études rurales, juillet-décembre, no 87-88, p. 295-308.

PICON, Bernard (1991) «Chasse, pêche, cueillette : un même objet support d'attitudes et de pratiques sociales différenciées", Sociétés contemporaines, $\mathrm{n}^{\circ} 8$, p. 87-100.

PORCHIER, J.-C. (2008) «Tourisme de pêche. Le miroir aux ablettes?» Espaces, $\mathrm{n}^{\circ}$ 260, juin, Pêche et Tourisme, p. 7-13.

RAIMBOURG, C. (2004) Étude de faisabilité pour le développement du tourisme pêche en Seine Maritime, mai-octobre, France : Comité départemental du tourisme de Seine Maritime. $142 \mathrm{p}$.

SOBRY, Claude (dir.) (2004) Tourisme sportif, Villeneuve d'Ascq : Presses Universitaires du Septentrion. $384 \mathrm{p}$.

SOL, Marie-Pierre (2007) «La patrimonialisation comme (re)mise en tourisme. De quelques modalités dans les "Pyrénées catalanes"», DANS VIOLIER, P. et O. LAZZAROTTI (dir.), Tourisme et patrimoine. Un moment du monde, p. 161-175. Saumur : Presses de l'Université d'Angers.

TOURAINE, Alain (1969) La société post-industrielle : Naissance d'une société, Paris : Bibliothèque Médiations. $319 \mathrm{p}$.

TRAIINI, Christophe (2004) «Territoires de chasse, territoires en questions», Revue Ethnologie française, ${ }^{\circ}$ 1, p. 41-48.

Union nationale des fédérations départementales des chasseurs $(1988,1994)$ Profils des chasseurs, Étude interne, Union nationale des fédérations départementales des chasseurs. 